\title{
Diagnosis and Staging of Acute Pancreatitis on Multidetector Computed Tomography
}

\author{
Sameera Vakil $^{1}$, Hina Siddiqui ${ }^{1}$, Maria Hassan ${ }^{2}$ \\ ${ }^{1}$ Department of Radiology, Dow University of Health Sciences, Karachi, Pakistan \\ ${ }^{2}$ Department of Radiology, Dr Ziauddin Hospital, Karachi, Pakistan
}

Email address:

sameera.vakil@gmail.com (S. Vakil), hinaa1085@gmail.com (H. Siddiqui), misshassan07@hotmail.com (M. Hassan)

\section{To cite this article:}

Sameera Vakil, Hina Siddiqui, Maria Hassan. Diagnosis and Staging of Acute Pancreatitis on Multidetector Computed Tomography. International Journal of Chinese Medicine. Vol. 3, No. 3, 2019, pp. 41-47. doi: 10.11648/j.ijcm.20190303.12

Received: July 5, 2019; Accepted: August 7, 2019; Published: August 28, 2019

\begin{abstract}
INTRODUCTION: Acute pancreatitis is a protean disease with varying clinical findings from mild to severe. CT is the imaging modality of choice to help stage the severity of inflammatory processes, detect pancreatic necrosis and depict local complications. Objectives of this study was to evaluate the role of MDCT in diagnosis and staging of acute pancreatitis and to identify the complications of acute pancreatitis on CT scanning. MATERIAL AND METHODS: This was a descriptive study done from 26th September 2016 to 25th March 2017. 45 patients of age range 17-85 years (29 Male and 16 Female) were included in study. CT scans were assessed for pancreatic necrosis and its complications. Computed tomography severity index (CTSI) was calculated by Balthazar's grading + degree of necrosis points into mild, moderate and severe CTSI. RESULTS: 14 patients had mild, 14 had moderate and 17 patients had severe acute pancreatitis CTSI. Of 31 patients with pancreatic necrosis $26(83.8 \%)$ patients showed complications and $5(16.12 \%)$ patients were without complications. Of 14 patients without pancreatic necrosis only 5 (35.7\%) patients were with complications and $9(64 \%)$ were without complications. CONCLUSION: CT scan should be the investigation of choice if clinical diagnosis is acute pancreatitis and to assess the severity of disease to predict its course.
\end{abstract}

Keywords: Acute Pancreatitis, Multidetector Computed Tomography, Computed Tomography Severity Index (CTSI)

\section{Introduction}

Acute pancreatitis is an acute inflammatory process of the pancreas with variable clinical manifestations. These range in the degree of severity from mild abdominal pain to severe life-threatening disease associated with high morbidity and mortality. The mortality in severe acute pancreatitis has been reported to be around $10-20 \%$. [1] Mortality of acute pancreatitis is dependent on the development of potentially lethal complications that can coexist and occur at any time following an acute attack. [4]

Alcoholism and biliary tract disease account for $90 \%$ of all cases of acute pancreatitis which occurs most often in middle life. Gallstones are present in $35-60 \%$ of cases of pancreatitis, and about $5 \%$ of patients with gallstones develop pancreatitis. The male to female ratio is $1: 3$ with biliary tract disease, and 6:1 in alcoholics. [5]

Management of patients with acute pancreatitis is based on the early assessment of severity of disease. Initial staging is established on clinical and laboratory grounds and on the findings of contrast enhanced computed tomography (CT) imaging. [4]

Contrast enhanced CT is the imaging modality of choice to help stage the severity of inflammatory processes, detect pancreatic necrosis and depict local complications. [5]

The introduction of multi slice (MDCT) has revolutionized the field of computed tomography and has created new dimensions in temporal and spatial resolution in CT scanning. The combination of multi planar imaging (coronal, sagittal) combined with $3 \mathrm{D}$ volume rendering is ideal for defining the true extent of disease. Volume displays are especially valuable in defining the inter-relationship between pseudo cysts and adjacent organs, as well as in defining vascular complications ranging from pseudo aneurysms, venous thrombosis and/ or collateralization. [6]

The prognostic value of computed tomography (CT) in acute pancreatitis has been previously investigated mainly by 
correlating the presence and extent of peri pancreatic fluid collection with the clinical severity of the disease, development of complications, and death. [7-9] Balthazar showed that patients without peri pancreatic inflammation (grade A and B) have a mild uncomplicated clinical course, while patients with one or several peri pancreatic collections (grade D and E) often exhibit a protracted clinical illness and high frequency of abscesses and death. Grading system of Balthazar allows identification of a subgroup of patients with acute pancreatitis in whom most serious complications will occur. The shortcoming of this system is that within this subgroup some patients (54\% in his series) show spontaneous mortality related to acute pancreatitis. This study was undertaken to indicate the resolution of these fluid collections, whereas the other $46 \%$ of individuals who could not be identified develop complications.

Keeping in mind the morbidity and role and significance of computed tomography in the diagnosis and assessment of complications of acute pancreatitis.

\section{Material and Methods}

It was a descriptive study conducted at a tertiary care hospital in Karachi from 26th September 2016 to 25th March 2017. All patients referred to the department for suspected acute pancreatitis during this period, were included in the study. Patients with absolute contrast media contraindication and pregnant patients were excluded from the study.

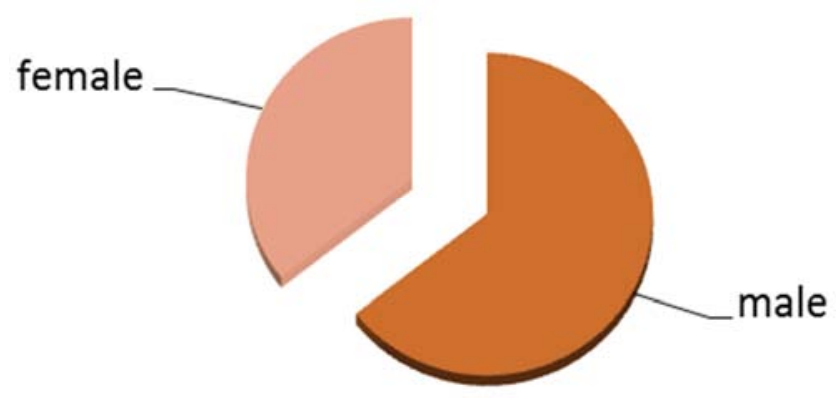

Figure 1. Sex Distribution Male $=29$ Female $=16$.

Table 1. CT severity index of acute pancreatitis.

\begin{tabular}{ll}
\hline Balthazar's Grading of acute pancreatitis & 0 \\
A-Normal pancreas & 1 \\
B-Pancreatic enlargement alone & 2 \\
C-Inflammation confined to the pancreas and & 3 \\
peripancreatic fat & 4 \\
D-One pancreatic fluid collection & \\
E-Two or more fluid collection & 0 \\
Degree of pancreatic necrosis & 2 \\
No necrosis & 4 \\
Necrosis of one-third of pancreas & 6 \\
Necrosis of one-half of pancreas & \\
Necrosis of more than one-half of pancreas &
\end{tabular}

Index was calculated by grade and degree of necrosis points. Patients were divided in to three categories: mild (0-3 points), moderate (4-6 points) and severe (7-10 points). For instance, a patient with CT grade D is assigned three points, if in addition, patient has more than $50 \%$ pancreatic necrosis, an additional six points are assigned, for a total index score of 9 .

Data analysis was done on SPSS (version 20). Data was validated by double entry.

It was done to describe each variable. It was performed by running frequency of all categorical and continuous variables. Measures of central tendencies (mean, median and mode), measures of dispersion (range and standard deviation) were performed for all continuous variables.

\section{Results}

Of 45 patients in the study, 14 (31.1\%) patients had mild pancreatitis (CTSI within range of $0-3$ ),

$14(31.1 \%)$ patients had moderate pancreatitis (CTSI within range of 4-6), and 17 (37.7\%) patients had severe acute pancreatitis (CTSI range of 7-10).

Of 45 patients, $16(35 \%)$ patients were below 40 years, 19 (42\%) were between 40-60 years and $10(22 \%)$ patients were above 60years.

$25(55 \%)$ patients were presented with epigastric pain, 20 (44\%) patients with generalized abdominal pain, 30 (66\%) patients in addition to abdominal pain had nausea and vomiting and $4(8.8 \%)$ patients had jaundice.

$21(46 \%)$ patients had history of cholelithiasis, $4(8 \%)$ patients had history of alcohol and $20(44 \%)$ patients had no known cause.

Serum amylase level was raised in all patients.

The relationship between CT severity index (CTSI) and age of patients and complications is summarized in table 2 .

Table 2. Correlation of CTSI with Age and Complications.

\begin{tabular}{|c|c|c|c|c|c|}
\hline \multirow[b]{2}{*}{ CTSI } & \multirow{2}{*}{$\begin{array}{l}\text { NUMBER } \\
\text { OF } \\
\text { PATIENTS }\end{array}$} & \multicolumn{3}{|c|}{ AGE (Years) } & \multirow{2}{*}{$\begin{array}{l}\text { COMPLICATION } \\
\text { (NUMBER OF } \\
\text { PATIENTS) } \\
\end{array}$} \\
\hline & & $<40$ & $40-60$ & $>60$ & \\
\hline MILD & 14 & 11 & 3 & 0 & 2 \\
\hline MODERATE & 14 & 3 & 7 & 4 & 12 \\
\hline SEVERE & 17 & 2 & 9 & 6 & 17 \\
\hline
\end{tabular}

There is good correlation between age of the patients and CTSI and complication. As the age of patients is increase the probability of severity of pancreatitis and complication will increase.

Pancreatic necrosis

The correlation between CTSI and degree of pancreatic necrosis is summarized in Table 3 .

Table 3. Correlation of CTSI with Degree of Necrosis.

\begin{tabular}{llll}
\hline \multirow{2}{*}{ CTSI } & \multicolumn{3}{l}{ PANCREATIC NECROSIS } \\
\cline { 2 - 4 } & ONE-THIRD & ONE-HALF & >ONE-HALF \\
\hline MILD & 3 & 0 & 0 \\
MODERATE & 10 & 1 & 0 \\
SEVERE & 0 & 8 & 9 \\
TOTAL & 13 & 9 & 9 \\
\hline
\end{tabular}

On the basis of CT assessment of pancreatic necrosis was detected in 31 patients. It was present in $3(21.4 \%)$ patients with one-third necrosis in mild acute pancreatitis. We have 
not found any patient of mild CTSI with half or more than half necrosis of pancreas.

In moderate acute pancreatitis all patients had pancreatic necrosis in which $10(71.4 \%)$ patients had one-third necrosis, $1(7.14 \%)$ patients had one-half necrosis and no one had more than one-half necrosis.

In severe acute pancreatitis, according to CTSI, total 17 patients had pancreatic necrosis in which $8(47 \%)$ patients with one-half necrosis and $9(53 \%)$ patients with more than one-half of pancreatic necrosis and no patient had one-third necrosis. $11(78.5 \%)$ patients with mild CTSI have not had any pancreatic necrosis.

Interesting results are come up when we correlate age of patients and pancreatic necrosis. It is summarized in Table 4.

Table 4. Correlation of Age with Degree of Necrosis.

\begin{tabular}{lllll}
\hline \multirow{2}{*}{ AGE (Years) } & \multicolumn{2}{l}{ DEGREE OF PANCREATIC NECROSIS } & \multirow{2}{*}{ NO PANCREATIC NECROSIS } \\
\cline { 2 - 5 } & ONE-THIRD & ONE-HALF & >ONE-HALF & 9 \\
\hline Less than 40 years & 2 & 3 & 2 & 5 \\
Between 40-60 years & 6 & 4 & 4 & 0 \\
More than 60 years & 5 & 2 & 3 & 14 \\
TOTAL & 13 & 9 & 9 & \\
\hline
\end{tabular}

Of 16 patients with age group of less than 40 years, 9 $(56.25 \%)$ patients were without pancreatic necrosis, 2 (12.5\%) patients with one-third necrosis, $3(18.75 \%)$ patients with one-half necrosis and $2(12.5 \%)$ patient had more than one-half necrosis of pancreas.

There were 19 patients in age group of 40-60 year, among them $6(31.5 \%)$ patients with one-third necrosis, $4(21 \%)$ patients with one-half necrosis and $4(21 \%)$ patients with more than one-half necrosis. $5(26 \%)$ patients have not had pancreatic necrosis.

There were 10 Patients who were above 60 years of age, 5 (50\%) patients had one-third necrosis, $2(20 \%)$ patient had one-half necrosis and $3(30 \%)$ patients had more than onehalf pancreatic necrosis.
The cause of pancreatic necrosis did not correlate well with presence and degree of pancreatic necrosis.

Of 31 patients with pancreatic necrosis $26(83.8 \%)$ patients were showed pancreatic and extra-pancreatic complications and $5(16.12 \%)$ patients were without complications.

Of 14 patients without pancreatic necrosis only $5(35.7 \%)$ patients were with complications and 9 (64\%) were without complications.

There is good correlation between age of patients and pancreatic and extra-pancreatic complications. Patients with more than 60 years of age had $90 \%$ complications as compare to patients less than 40 years of age, they only had $56 \%$ of complications. Values are summarized in Table 5.

Table 5. Correlation of Age with Complications.

\begin{tabular}{lll}
\hline \multirow{2}{*}{ AGE (YEARS) } & COMPLICATIONS & \\
\cline { 2 - 3 } & PRESENT & ABSENT \\
\hline$<40$ & $9(56 \%)$ & $7(44 \%)$ \\
$40-59$ & $13(68 \%)$ & $6(32 \%)$ \\
$>60$ & $9(90 \%)$ & $1(10 \%)$ \\
\hline
\end{tabular}

Among 31 patients with complications, 2 patients had mild CTSI, 12 patients with moderate CTSI and 17 patients with severe CTSI.

Of 2 patients with mild CTSI, both patients had acute pancreatic fluid collection, peripancreatic and mesenteric inflammation. 3 patients had swollen pancreas with peri-renal fluid collection and peripancreatic inflammation. One patient had only peripancreatic inflammation, mesenteric and perirenal fat stranding.

Of 12 patients with moderate CTSI, all patients had pancreatic necrosis and peripancreatic inflammation.

Of 12 patients, 3 patients had acute pancreatic fluid collection, 3 patients had pseudocyst among these three, one patient had splenic vein thrombosis, of 12 patients 4 patients had pancreatic abscess and 4 had pleural effusion.

Of 17 patients with severe CTSI, all patients had pancreatic necrosis with peripancreatic inflammation, mesenteric inflammation, perirenal fat stranding and pleural effusion.

One patient with pancreatic abscess had splenic artery pseudoaneurysm.5 patients had acute perirenal fluid collection, 8 patients had pancreatic abscess and 7 patients with pseudocyst, among these 4 patients 3 had duodenal wall thickening.

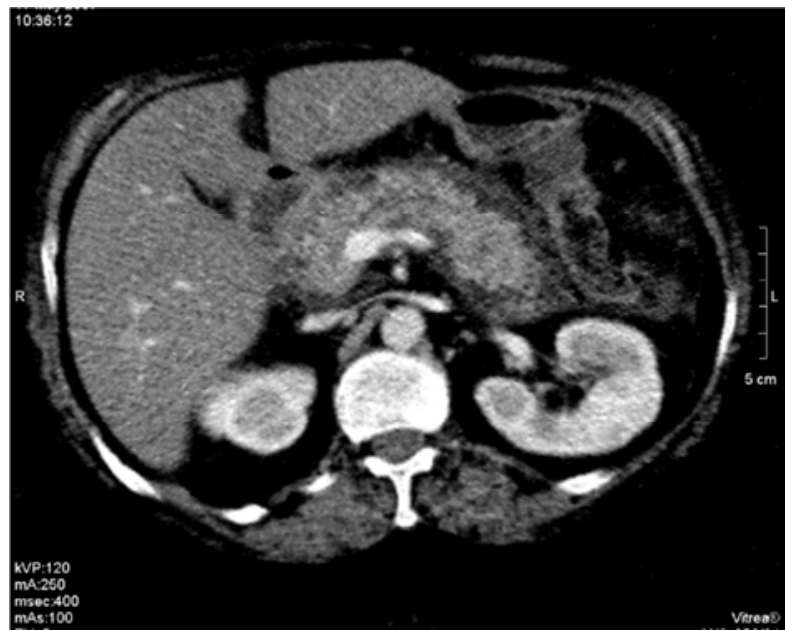

Figure 2. 62 yrs old patient with pancreatic swelling and enlargement and peripancreatic collection tracking into lesser sac and left pararenal space. 


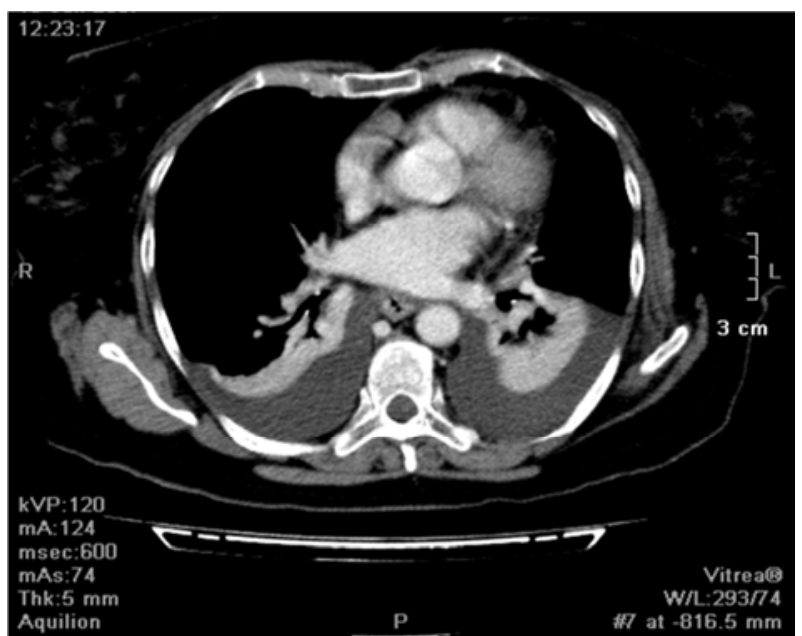

(a)

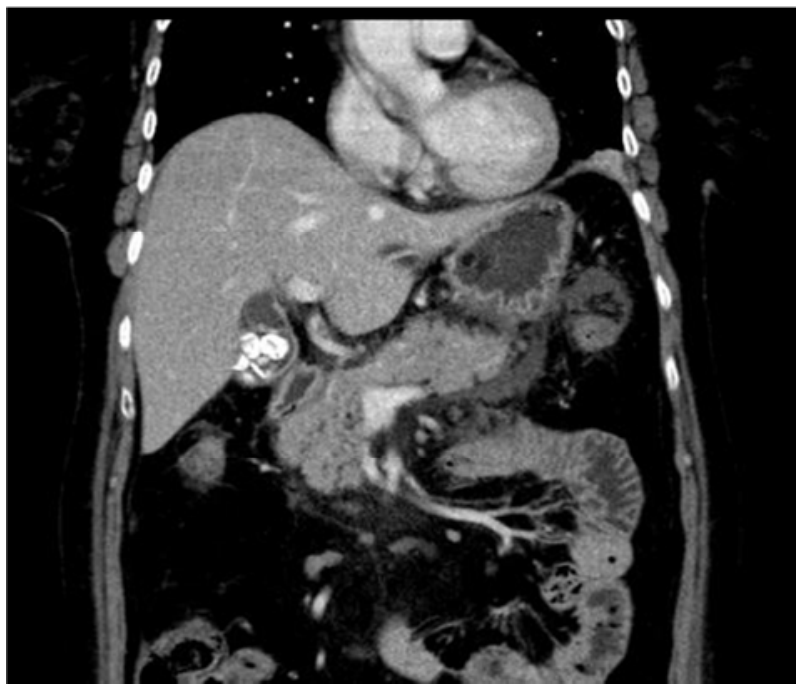

(b)

Figure 3. (a) Bilateral pleural effusion and (b) multiple Gall stones in a patient with acute pancreatitis.

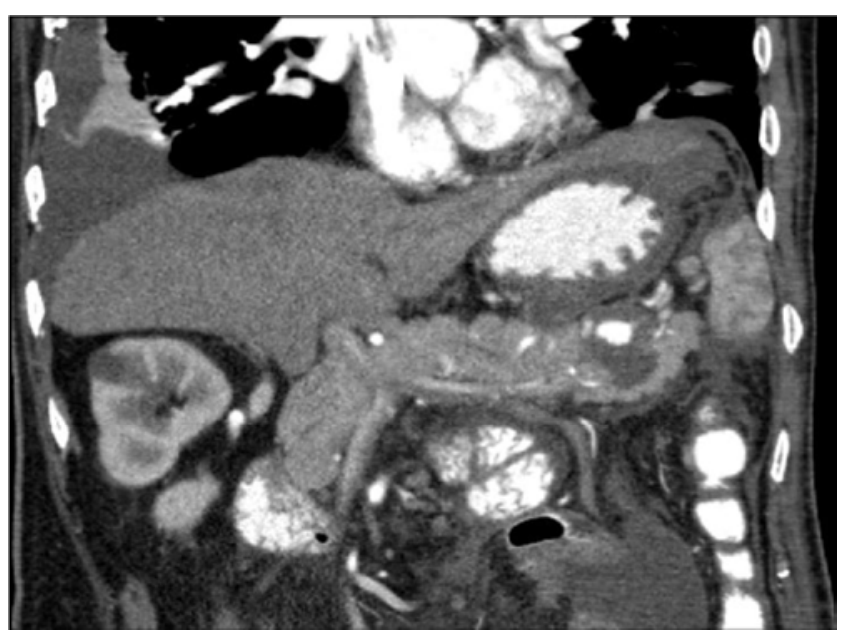

Figure 4. Curved MPR images of 66 yrs old male showing cystic lesion in the tail of pancreas representing pseudocyst formation there is also aneurysmal dilatation of the splenic artery projecting into the pancreatic pseudocyst.

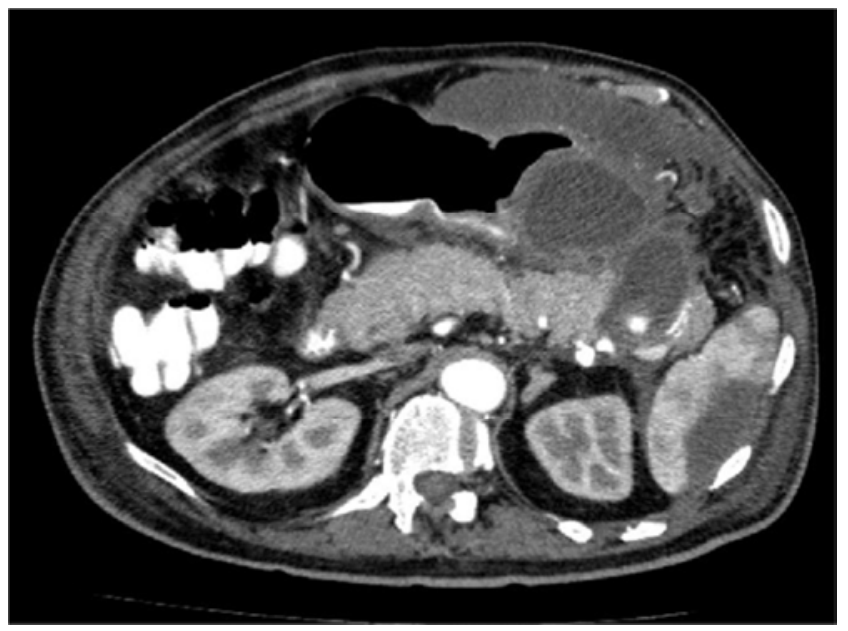

Figure 5. Axial image of $66 \mathrm{yrs}$ old male showing cystic lesion in the tail of pancreas representing pseudocyst formation there is also aneurysmal dilatation of the splenic artery projecting into the pancreatic pseudocyst.

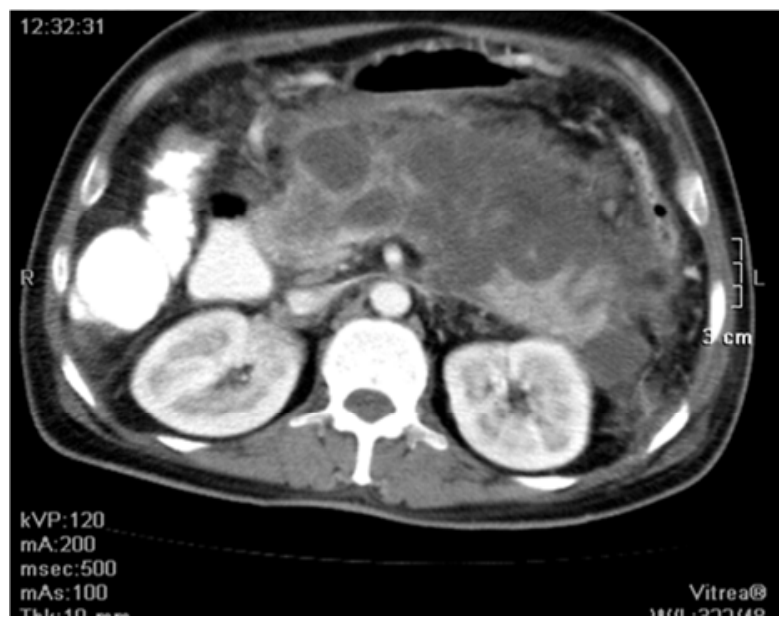

(a)

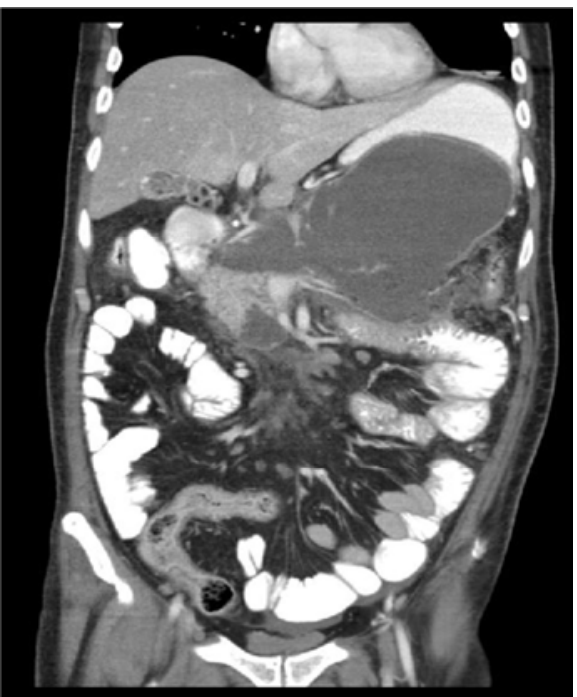

(b)

Figure 6. Large cystic mass in region of head, body and tail of pancreas largely replacing the pancreas with presence of septae within it representing pancreatic pseudocyst. 


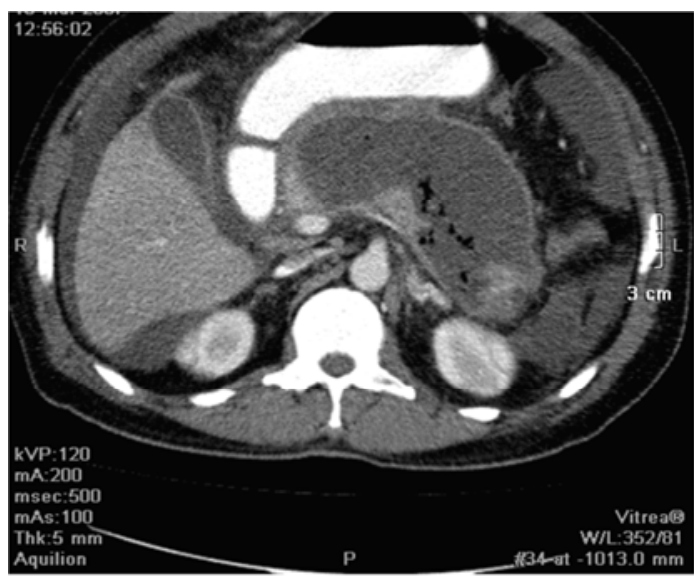

(a)

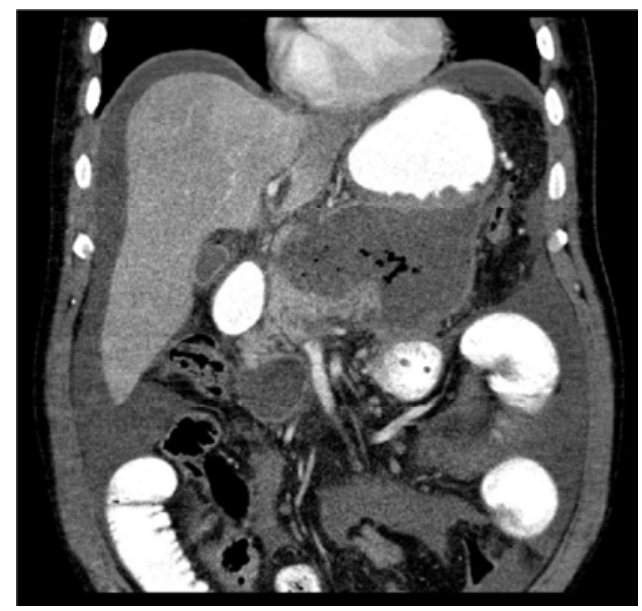

(b)

Figure 7. (a) Axial and (b) coronal reconstructed images of 50 yrs old male with presence of a large abscess in the body and tail of pancreas, it shows peripheral rim of contrast enhancement and multiple pockets of air lucencies within it.

\section{Discussion}

Acute pancreatitis is a reversible inflammatory process of the pancreas. Although the disease process may be limited to pancreatic tissue, it also can involve peripancreatic tissues or more distant organ sites. Acute pancreatitis may occur as an isolated attack or may be recurrent. It has a variety of causes and can range in severity from mild to severe and life threatening. Some patients may require brief hospitalization, whereas others may be critically ill with multiple organ dysfunction requiring intensive care monitoring. Mild acute pancreatitis has a very low mortality rate (less than $1 \%$ ), [1011] whereas the death rate for severe acute pancreatitis can be 10 to $30 \%$ depending on the presence of sterile versus infected necrosis. [11]

So detection of pancreatic necrosis is very important in patients with acute pancreatitis.

A highly accurate system which could predict the severity and identify the local extent and complications of a serious inflammation, is beneficial for patient outcome. [12] An ideal or desirable detection system should have high sensitivity and positive predictive value. It should be able to detect necrosis early in the course of disease. It should be rapidly performed and available in most hospitals.

Of all the available means for assessing the disease severity, only CT scan can reliably detect the pancreatic necrosis and meets almost all have above mentioned criteria.

No single laboratory or clinical sign is pathognomonic for acute pancreatitis; many biomarkers and inflammatory mediators for predicting the severity of acute pancreatitis are being evaluated. The initial laboratory evaluation should include amylase and lipase levels; complete blood count with differential; metabolic panel (blood ureanitrogen, creatinine, glucose, and calcium levels); triglyceride level; urinalysis; and arterial blood gases. [13]

Amylase and lipase, secreted by the acinar cells of the pancreas, are the most common laboratory markers used to establish the diagnosis of acute pancreatitis. [14] Elevated amylase and lipase levels can be nonspecific, depending on the time since onset of pain, other intra-abdominal processes, and concomitant chronic diseases such as renal insufficiency. [15-16] Amylase levels may be normal in patients with alcoholism who present with acute pancreatitis, especially if they have had previous attacks of alcoholic pancreatitis; thus, serial testing may not be helpful. Plasma lipase is more sensitive and specific than plasma amylase. 16

Recent research has examined potential biologic markers for predicting the severity and prognosis of pancreatitis. Trypsinogens and pancreatic proteases involved in the autodigestive processes of acute pancreatitis appear promising. Other investigational serologic markers include trypsinogen activation peptide, C-reactive protein, procalcitonin, phospholipase $\mathrm{A} 2$, and the cytokines interleukin-6 and interleukin-8. [16-19] Currently, these markers have limited clinical availability, but there is significant interest in better understanding markers of immune response and pancreatic injury because these could be valuable tools for reliably predicting the severity of acute pancreatitis and supplementing imaging modalities. [20, 21]

Aspiration of intraperitoneal fluid and peritoneal lavage has been used as an indicator of severity of pancreatitis. Aspiration of $10-20 \mathrm{ml}$ of peritoneal fluid or dark color of lavaged fluid is often seen in patients with severe pancreatitis. But its sensitivity is low and its an invasive procedure with complications and not well tolerated by the patients. Several scoring systems can predict the severity of pancreatitis, and recent work has attempted to compare their relative predictive values.

Ranson's criteria, the Acute Physiology and Chronic Health Evaluation (APACHE II) scale, and the Computed Tomography (CT) Severity Index have been developed and validated to predict adverse outcomes, including mortality, in patients with pancreatitis. [20]

The APACHE II system allows monitoring of disease progression and response to therapy but the system is complex, more difficult to perform and is less accurate for identification of local complications. [22]

Conventional abdominal X-rays, barium studies and chest 
X-rays show indirect signs of pancreatitis but have only limited role in the early evaluation of disease severity. Ultrasound is helpful in identifying gallstones and common duct stones. However, it is not sensitive in the early detection of pancreatic necrosis. In addition, ultrasound has its limitations because visualization of pancreas is often impaired because of overlying bowel gases. [8, 23] A diffusely enlarged and hypoechoic gland is consistent with interstitial edema, while an extrapancreatic fluid collections in the lesser sac and anterior pararenal space can be detected in patients with severe disease. CT scan is sensitive in the detection of early pancreatic necrosis. Pancreatic gland necrosis is a diffuse or local area of nonviable pancreatic parenchyma that typically is associated with peripancreatic fat necrosis. Normal unenhanced pancreas has CT attenuation of 30-50 HFU and shows homogeneous enhancement with post contrast attenuation of 100-150 HFU. [2, 17] A focal or diffuse well-marginated zone of un-enhanced pancreas, larger than $3 \mathrm{~cm}$ in diameter or larger than $30 \%$ of the area of pancreas, is considered a reliable CT finding for diagnosis of necrosis. CT is $80-90 \%$ accurate in the detection of pancreatic necrosis. Specificity of CT increases with increasing percentage of pancreatic necrosis. Specificity of $\mathrm{CT}$ is about $50 \%$, if there are only small areas of necrosis however, in more than $30 \%$ necrosis, specificity of CT is $100 \%$. In addition to early detection of pancreatic necrosis, there are other CT staging criteria of acute pancreatitis. These include grades of acute pancreatitis. There are 5 grades of acute pancreatitis from A to E. CTSI or CT severity index of acute pancreatitis is then calculated from grade of acute pancreatitis and degree of pancreatic necrosis. [24]

We have calculated CTSI and correlated it with degree of pancreatic necrosis.

We have found good correlation between mild, moderate and severe acute pancreatitis and degree of pancreatic necrosis. This has been shown in table 3. Complications have also correlation with CTSI. Complications developed in 2 patients with mild pancreatitis, 12 patients with moderate pancreatitis and 17 patients with severe pancreatitis. We also correlated CTSI and degree of necrosis with age. There is a good correlation between severity of pancreatitis and age and degree of pancreatic necrosis and age. These has been shows in table 2 and table 4 . No statistically significant correlation is seen in development of late complications with age. This has been shown in table 5. CT has also its role in the management of patients with acute pancreatitis in addition to diagnosis and assessment of disease severity and assessing prognosis. [3] CT along with ultrasound can be used for percutaneous drainage procedures. [1, 2] CT with multiplanar reconstruction is valuable in helping determine the optimal pathway for pseudocyst drainage. Procedures such as cystgastrostomy can be planned off the sagittal and 3D volume mapping. CT however, provides more information about the extent, number of peripancreatic collections and location of adjacent structures. It is also much better seen with CT scan, compared to ultrasound. [18]

In the previously available studies and literature in diagnosis of acute pancreatitis, evaluation of severity of disease and development of complications have been studied. However, previously all these findings were evaluated separately. We have evaluated all these findings collectively in our study.

In addition, we have correlated severity of pancreatitis and degree of pancreatic necrosis with age.

\section{Conclusion}

Severity of acute pancreatitis is assessed by clinical and laboratory evaluation and contrast enhanced CT scan. Numeric systems like Ranson's criteria and APACHE2 are commonly used to help detect organ failure. Clinical and laboratory evaluations are not sensitive in detecting pancreatic necrosis. Numeric systems only induct correlation with disease severity with a low sensitivity of about $70 \%$. Contrast enhanced CT scan is imaging modality of choice in the staging of severity of acute pancreatitis. Detection of pancreatic necrosis as prognostic indicator to depict local complications. CT scan has overall sensitivity of $90 \%$ in the detection of pancreatic necrosis and is about $100 \%$ sensitive after 4 days in detecting pancreatic necrosis. CT severity index has excellent correlation with development of local complications and incidence of death in patients with acute pancreatitis. Although most severe complications developed in patients with peripancreatic collections and some of these patients die, such complications occur mostly in individuals with associated early or late pancreatic necrosis. Most individual fluid collections tend to resolve spontaneously, if pancreas maintains its integrity and there is no necrosis.

The introduction of multislice (MSCT) has revolutionized the field of computed tomography and has created new dimensions in temporal and spatial resolution in CT scanning. the combination of multiplanar imaging (coronal, sagittal) combined with $3 \mathrm{D}$ volume rendering is ideal for defining the true extent of disease. Volume displays are especially valuable in defining the inter-relationship between pseudocysts and adjacent organs, as well as in defining vascular complications ranging from pseudoaneurysms, venous thrombosis and/ or collateralization.

The limitations of early CT examination are related to potential development of complications in patients with normal pancreas. For this reason, patients with peripancreatic fluid collection with no visible pancreatic necrosis should undergo CT scan every two weeks or even earlier if there is clinical indication. Detection of pancreatic necrosis either at first examination or at follow up study or a high CTSI at initial examination are indicators of severe pancreatitis that enables the radiologist to identify a group of patients in whom most life threatening complications will occur. Pancreatitis tends to be more severe in older patients and average hospital is more in patients with severe pancreatitis compared to mild pancreatitis.

We can say that CT scan should be the investigation of choice if clinical diagnosis is acute pancreatitis and we want to assess the severity of acute pancreatitis to predict the 
course of disease.

\section{References}

[1] Bader HA, Abd El Azeem AS. Role of computed tomography in diagnosis, follow-up, and minimally invasive treatment of acute pancreatitis. Sci J Al-Azhar Med Fac Girls 2018; 2: 527.

[2] Lim YY, O'Shea S, Lee SH. Diagnostic and interventional radiology workload in acute pancreatitis in an ITU/HDU setting. Clinical Radiology 2006; 61: 86-90.

[3] Jiruppabha B. CT appearance of acute pancreatitis using multiphase Multidetector Computed Tomography and correlation between CT Severity Index and clinical outcomes. Chula Med J Vol. 63 No. 3 July - September 2019; 153-161.

[4] Balthazar EJ. Complications of acute pancreatitis: clinical and CT evaluation. Radiol Clin North Am 2002; 40: 1211-1227.

[5] Chishty IA, Bari V, PashaS, BurhanD, HaiderZ, Rafique Z. Role of computed tomography in acute pancreatitis and its complications among age groups. J Pak Med Assoc 2005; 10: 431-435.

[6] Balthazar EJ. Staging of acute pancreatitis. Radiol Clin North Am 2002; 40: 1199-1209.

[7] Aschoff AJ, Gessel RV, BrambsHJ, Merkle EM. Effect of iodine concentration of contrast media on contrast enhancement in multislice CT of the pancreas. British Journal of Radiology 2004; 77: 821-830.

[8] Johnson CD. Pancreatic carcinoma: developing a protocol for multidetector row CT. Radiology 2001; 220: 3-4.

[9] Mortele KJ, Mergo PJ, Taylor HM, Ernst MD, Ros PR. Renal and perirenal space involvement in acute pancreatitis: Spiral CT findings. Abdominal Imaging 2000; 25: 272-8.

[10] Mortele KJ, Mergo PJ, Taylor HM, Ernst MD, Ros PR. Splenic and perisplenic involvement in acute pancreatitis: Determination of prevalence and morphologic helical CT features. J Computer Assisted Tomography 2001; 25: 50-4.

[11] Tsushima Y, Tamura T, Tomioka K, Okada C, Kusano S, Endo K. Transient splenomegaly in acute pancreatitis. BJR 1999; 72: 637-43.

[12] Russo MW, Wei JT, Thiny MT, Gangarosa LM, Brown A, Ringel Y, et al. Digestive and liver diseases statistics, 2004. Gastroenterology 2004; 126: 1448-53.
[13] Triester SL, Kowdley KV. Prognostic factors in acute pancreatitis. J Clin Gastroenterol 2002; 34: 167-76.

[14] Dervenis C, Johnson CD, Bassi C, Bradley E, Imrie CW, McMahon MJ, et al. Diagnosis, objective assessment of severity, and management of acute pancreatitis. Santorini consensus conference. Int J Pancreatol 1999; 25: 195-210.

[15] Gürleyik G, Emir S, Kiliçoglu G, Arman A, Saglam A. CTSI, APACHE II score and serum CRP concentration for predicting the severity of acute pancreatitis. JOP 2005 Nov 10; 6 (6): 562-7.

[16] Society for Surgery of the Alimentary Tract (SSAT). Treatment of acute pancreatitis. Manchester, Mass.: Society for Surgery of the Alimentary Tract (SSAT), 2004. Accessed December 8 2006, athttp//www.guidelines.gov/summary/summary.aspx?view id $=1 \& d o c \_i d=5512 \# \mathrm{~s} 25$.

[17] Mitchell RM, Byrne MF, Baillie J. Pancreatitis. Lancet 2003; 361: $1447-55$

[18] Smotkin J, Tenner S. Laboratory diagnostic tests in acute pancreatitis. J Clin Gastroenterol 2002; 34: 459-62.

[19] Frossard JL, Hadengue A, Pastor CM. New serum markers for the detection of severe acute pancreatitis in humans. Am J Respir Crit Care Med 2001; 164: 162-70.

[20] Neoptolemos JP, Kemppainen EA, Mayer JM, Fitzpatrick JM, Raraty MG, Slavin J, et al. Early prediction of severity in acute pancreatitis by urinary trypsinogen activation peptide: a multicentre study. Lancet 2000; 355: 1955-60.

[21] Tenner S. Initial management of acute pancreatitis: critical issues during the first 72 hours. Am J Gastroenterol 2004; 99: 2489-94.

[22] Balthazar EJ, Robinson DL, Megibow AJ, Ranson JH. Acute pancreatitis: value of CT in establishing prognosis. Radiology 1990; 174: 331-6.

[23] Robert JH, Frossard JL, Mermillod B, Soravia C, Mensi N, Roth M, et al. Early prediction of acute pancreatitis: prospective study comparing computed tomography scans, Ranson, Glasgow, acute physiology and chronic health evaluation II scores and various serum markers. World J Surg 2002; 26: 612-9.

[24] Simchuk EJ, Traveso LW, Nukui Y, Kozarek RA. Computed tomography severity index is a predictor of outcomes for severe pancreatitis. Am J Surg 2000; 179: 352-5. 